\title{
Zoledronic Acid Elicits Proinflammatory Cytokine Profile in Osteolytic Prostate Cancer Cells
}

\author{
Yi-Chia Lin, ${ }^{1,2}$ Po-Cheng Liao, ${ }^{3}$ Te-Fu Tsai, ${ }^{1,2}$ Kuang-Yu Chou, ${ }^{1,2}$ Hung-En Chen, \\ Ji-Fan Lin, ${ }^{3}$ and Thomas I-Sheng Hwang ${ }^{1,2,4}$ \\ ${ }^{1}$ Division of Urology, Department of Surgery, Shin-Kong Wu Ho-Su Memorial Hospital, Taipei 11101, Taiwan \\ ${ }^{2}$ Division of Urology, School of Medicine, Fu-Jen Catholic University, New Taipei City 24205, Taiwan \\ ${ }^{3}$ Central Laboratory, Shin-Kong Wu Ho-Su Memorial Hospital, Taipei 11101, Taiwan \\ ${ }^{4}$ Department of Urology, Taipei Medical University, Taipei 11031, Taiwan
}

Correspondence should be addressed to Thomas I-Sheng Hwang; m001009@ms.skh.org.tw

Received 24 January 2014; Accepted 12 March 2014; Published 23 April 2014

Academic Editors: A. Ozcan and Y. Wang

Copyright (c) 2014 Yi-Chia Lin et al. This is an open access article distributed under the Creative Commons Attribution License, which permits unrestricted use, distribution, and reproduction in any medium, provided the original work is properly cited.

\begin{abstract}
Zoledronic acid (ZA), a bisphosphonate used to prevent skeletal fractures in patients with cancers, was demonstrated to induce apoptosis in a number of cancer cells. Our previous study showed that ZA also induces autophagic cell death in metastatic prostate cancer cells. However, the clinical trials using ZA in the treatment of metastatic prostate cancer did not have a longer diseases-free period. Since most of ZA was attracted to the bone after administration, we hypothesized that local prostate cancer cells may evolve prosurvival pathways upon low concentration of ZA treatment. In this study, we investigated the inflammatory effects of ZA on osteolytic PC3 prostate cancer cell, since inflammation was reported to be related to cancer development and survival. Exposure of PC3 cells to various concentrations of ZA resulted in induction of apoptosis and autophagy. The expression of inflammatory biomarkers including interleukin 6 (IL-6), cyclooxygenase-2 (COX-2), and NF- $\kappa$ B was remarkably upregulated in response to ZA treatment in a dose- and time-dependent manner. The production of IL- 6 was elevated upon ZA treatment. The antiapoptotic protein $\mathrm{Bcl} 2$ was increased with parallel increased level of IL-6. Our data suggest that treatment with low concentrations of ZA enhances the inflammatory profile and may serve as a prosurvival signaling pathway in PC3 cells.
\end{abstract}

\section{Introduction}

Zoledronic acid (ZA), a nitrogen-containing bisphosphonate, is predominately prescribed to cancer patients with bone resorptive diseases owing to metastasis. ZA possesses great bone affinity and exerts interference effect on osteoclasts functions through inhibition of protein prenylation and induction of apoptosis $[1,2]$. Since zoledronic acid is recognized to significantly decrease skeletal-related events in prostate cancer patients with bony metastasis, the medication has been suggested to be a standard treatment modality for patient in this group. In addition to decreasing skeletalrelated events, ZA has been reported to directly or indirectly interfere with cancer progression and tumor growth in the cellular level [3].
Mechanisms by which ZA directly inhibits cancer growth include activation of autophagy and induction of apoptosis. Indirect anticancer effects of ZA involve activation of immune responses against cancer, inhibition of angiogenesis, and interference of cell-cell interactions with mesenchymal stem cells. Recent studies have demonstrated that ZA possesses immunomodulatory activity [4-6]. A survey-based study reveals that patients receiving ZA exhibit altered inflammatory profiles [7]. The treatment with ZA is suggested to contribute to inflammatory phenotype by recruiting and activating macrophage with results of enhanced cytokine production [8-10]. However, the effect of ZA on cancer cells in the aspect of inflammatory response remains sketchy. Therefore, we conducted a study with the hypothesis that osteolytic prostate cells (PC3) respond to ZA treatment by 

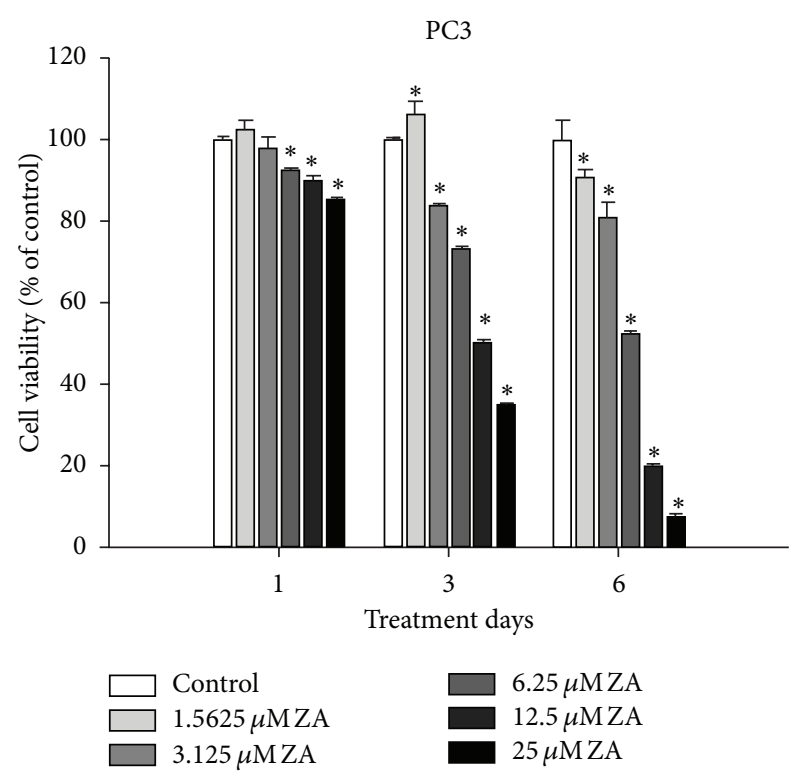

FIgURE 1: Effect of ZA on cell viability of PC3 cells. PC3 cells were treated with ZA for 1, 3, and 6 days. Cell viability was determined by MTT assay. The data represent the mean \pm SD of three independent experiments. ${ }^{*} P<0.05$ compared with the ZA-untreated control cells.

upregulating expressions of levels of inflammatory biomarkers such as IL-6, COX-2, IL- $1 \beta$, and NF- $\kappa$ B.

\section{Materials and Methods}

2.1. Cell Culture. Human prostate cancer cell line PC3 (CRL1435) obtained from the American Type Culture Collection (ATCC, Rockville, MD, USA) was used in this study. Cells were maintained in Dulbecco's modified Eagle's medium (DMEM, Invitrogen, CA, USA) supplemented with $10 \%$ fetal bovine serum (FBS, Invitrogen) and $1 \%$ antibiotics $(10,000$ unit $/ \mathrm{mL}$ penicillin, $10 \mathrm{mg} / \mathrm{mL}$ streptomycin, and $0.025 \mathrm{mg} / \mathrm{mL}$ amphotericin) in a humidified atmosphere of $5 \% \mathrm{CO}_{2}$ at $37^{\circ} \mathrm{C}$. Cells were seeded and incubated overnight prior to treatment.

2.2. Cell Viability Assay. Cell viability was determined using MTT assay. Cells were seeded in 96-well plate at a density of $1 \times 10^{4}$ cells/well overnight prior to treatment. After treatment, the medium was replaced with $0.5 \mathrm{mg} / \mathrm{mL} \mathrm{3-}$ (4,5-dimethylthiazol-2-yl)-2,5-diphenyltetrazolium bromide (MTT, Sigma-Aldrich, MO, USA) solution. After 3 hours of incubation at $37^{\circ} \mathrm{C}$, the supernatant was removed and subsequently dimethyl sulfoxide (DMSO, Sigma-Aldrich) was added to release formazan. The resulting absorbance was measured at a wavelength of $540 \mathrm{~nm}$ with background subtraction at $650 \mathrm{~nm}$ on EMax Endpoint ELISA Microplate Reader (Molecular Devices Inc., CA, USA).

2.3. Real-Time PCR. Total RNA was extracted using TRIzol reagent (Invitrogen). The amount of extracted RNA was measured by Qubit RNA Assay Kit (Invitrogen). Reverse transcription was performed before real-time PCR amplification by high capacity cDNA reverse transcription kits (Invitrogen). Relative expression of target genes was assessed by ABI 7900HT system (Applied Biosystems, CA, USA). The housekeeping gene, GAPDH, was used as an internal control. The primer sequences are listed in Table 1.

2.4. Detection of IL-6 Production by ELISA. Cells were treated with $\mathrm{ZA}$ at indicated concentrations and the culture medium was collected to measure IL- 6 secretion from cells using human IL-6 screening set (Thermo, IL, USA). Culture medium $(100 \mu \mathrm{L})$ was loaded in precoated 96-well plate and incubated for 1 hour at room temperature. The plate was washed by phosphate-buffered saline with Tween (PBST) twice. Secondary antibody was incubated for 1 hour at room temperature. Subsequently, streptavidin-HRP was incubated for 30 minutes at room temperature. TMB substrate was used to develop in dark for 30 minutes and O.D. 650 was measured on an ELISA reader (Wave X-340, Bio-Tek instruments, Inc., VT, USA).

2.5. Western Blotting. Cells were washed by PBS twice and incubated with RIPA buffer (Sigma-Aldrich) on ice for $5 \mathrm{~min}$ utes. Samples were centrifuged at $12,000 \mathrm{rpm}$ for 15 minutes at $4^{\circ} \mathrm{C}$. The supernatants were collected to perform western blot. The extracted protein concentration was measured by Qubit Protein Assay Kit (Invitrogen). About $20 \mu \mathrm{g}$ of protein samples were loaded in SDS-PAGE and then transferred to PVDF using $100 \mathrm{~V}$ for 1 hour at $4^{\circ} \mathrm{C}$. Blocking was performed with 5\% BSA in Tris-buffered saline with Tween (TBST). Membranes were incubated with rabbit anti-mouse LC3II, procaspase-3 (Cell Signaling Technology, Beverly, MA, USA), and $\beta$-actin (Sigma-Aldrich) for 1 hour at room temperature. The blots were washed by TBST and incubated with secondary antibody for 30 minutes at room temperature. The blots were developed using ECL system (Millipore, MA, USA).

2.6. Statistical Analysis. All experiments were performed at least in triplicate, and data are shown as the means \pm standard deviation (SD). The statistical significance of the difference in the measured variables between the treatment and control groups was determined by Student's $t$-test and was considered significant at $P<0.05$.

\section{Results}

3.1. Zoledronic Acid Inhibits Growth of PC3 Cells through Induction of Autophagy and Apoptosis. As ZA has been demonstrated to exert anticancer activity at high concentration, PC3 cells were treated with ZA at a series of concentrations $(0-25 \mu \mathrm{M})$ and the inhibitory effect on cancer proliferation was determined using MTT assay. As shown in Figure 1, ZA inhibited the growth of PC3 cells in both doseand time-dependent manners, with the greatest effect at 12.5 and $25 \mu \mathrm{M}$. Anticancer property of ZA has been reported to consist of apoptosis or autophagy [11, 12]. Our results showed 
TABLE 1: Primer list for real-time PCR.

\begin{tabular}{lll}
\hline Gene & Forward primer & Reverse primer \\
\hline COX-2 & TCCCTGAGCATCTACGGTTTG & TCTCCTATCAGTATTAGCCTGCTTGTC \\
IL-1 $\beta$ & TTACAGTGGCAATGAGGATGACTT & AGTGGTGGTCGGAGATTCGT \\
IL-6 & AGCCCTGAGAAAGGAGACATGTA & AGGCAAGTCTCCTCATTGAATCC \\
NF- $\kappa$ B & TGCTGGGATGAAGCATGGA & GATCTTGCTCTGTGGTTTCAATAACT \\
Bax & TGGAGCTGCAGAGGATGATTG & GGCCTTGAGCACCAGTTTG \\
Bcl2 & GCTATAACTGGAGAGTGCTGAAGATTG & TGATGTTGTATTTTTAAGTACAGCATGAT \\
ATG5 & ATGGACAGTTGCACACACTAGGA & ATCTTCAGGATCAATAGCAGAAGGA \\
Beclin 1 & CTCACAGCTCCATTACTTACCACAG & TCAATAAATGGCTCCTCTCCTGA \\
GAPDH & GGGTGTGAACCATGAGAAGTATGA & GGTGCAGGAGGCATTGCT \\
\hline
\end{tabular}

that exposure of PC3 cells to ZA led to a significant increase in mRNA expression of autophagy-related genes, ATG5 and Beclinel (Figures 2(a) and 2(b)). Autophagy induced by ZA treatment in PC3 cells was characterized by formation of autophagosomes [12]. Using western blot, the results revealed that LC3-II was accumulated upon ZA treatment in a dose- and time-dependent manner (Figure 2(c)). The trend of autophagy gene upregulation was consistent with that of the degrees of cell death in response to ZA treatment. In addition to autophagy genes, we observed a consistent trend toward increased mRNA expression of the proapoptotic protein Bax in response to ZA exposure (Figure 2(d)). The apoptotic activity of ZA was supported by decreased level of procaspase-3 using western blotting (Figure 2(e)).

\subsection{Inflammation-Associated Genes Were Upregulated in Zole-} dronic Acid-Treated PC3 Cells. We investigated whether ZA induces inflammatory responses in prostate cancer cells. The effects of ZA on expressions of inflammatory mediator in PC3 cells were evaluated. Using real-time PCR, mRNA expression of IL-6 in PC 3 cells was significantly increased in response to the presence of ZA in a dose- and time-dependent fashion (Figure 3(a)). COX-2 synthesizing an inflammatory mediator, prostaglandin E2, has been reported to be associated with increased malignant potential [13]. Hence, we examined the mRNA expression of COX-2 in PC3 cells in response to ZA treatment. ZA treatment at concentrations of 12.5 and $25 \mu \mathrm{M}$ significantly increased COX-2 mRNA expression levels. The trend of increased COX-2 expression was similar to that of IL-6 expression. The response to ZA exposure was dose- and time-dependent (Figure 3(b)). We also evaluated the effect of ZA on expression of IL-1 $\beta$ in PC 3 cell. Exposure to ZA for 72 hours caused insignificant change in IL- $1 \beta$ mRNA expression. Unexpectedly, PC3 cells treated with ZA for 6 days resulted in a significant decrease in IL-1 $\beta$ mRNA expression (Figure 3(c)).

3.3. Zoledronic Acid Enhanced IL-6 Production and Prosurvival Bcl2 Expression in PC3 Cells. The NF- $\kappa \mathrm{B}$ has been demonstrated to play a critical role in the regulation and production of a broad variety of proinflammatory mediator such as IL-6. To investigate whether NF- $\kappa$ B is involved in inflammatory effect of ZA, mRNA expression of NF- $\kappa$ B in
PC3 cells was examined. Results of real-time PCR revealed that expression of NF- $\kappa \mathrm{B}$ was upregulated in response to ZA exposure (Figure 4). We next investigated influence of ZA on IL-6 production in PC3 cell culture medium using ELISA. As shown in Figure 5, significant secretions of IL-6 were detected after exposure of PC3 cells to 12.5 and $25 \mu \mathrm{M}$ of ZA. The production of IL- 6 was 5 -fold higher than that of untreated cells 6 days after treatment. It is consistent with the trend in the upregulation of inflammatory mediator genes aforementioned. We next determined the mRNA expression of $\mathrm{Bcl} 2$, a survival protein that is regulated by IL-6. ZA treatment induced an increase in mRNA expression of $\mathrm{Bcl} 2$ in PC3 cells (Figure 6).

\section{Discussion}

Zoledronic acid is commonly prescribed to patients with malignant diseases accompanying bony metastasis including metastatic prostate cancer $[14,15]$. Increasing evidences have been reported to support anticancer effect of ZA, through the inhibition of cancer cell growth and interference of metastasis $[3,16]$. Despite documented safety and tolerability of ZA, adverse effects and complications have been reported including osteonecrosis of jaw and flu-like symptoms. Additionally, antitumor property of ZA is controversial and disputative in clinical setting. Hence, the effect of ZA on the modulation of inflammatory response in metastatic prostate cancer demands a reevaluation. In the present study, we found that exposure to ZA markedly upregulated mRNA expression of inflammatory biomarkers in osteolytic prostate cancer cells including IL-6 and COX-2. Moreover, secretion of IL- 6 by ZA-treated PC3 cells was increased in parallel to transcriptional degree of NF- $\kappa \mathrm{B}$, an upstream regulatory factor. Anticancer activity of ZA at various concentrations was observed through conclusively a synergic effect of apoptosis and autophagy.

Accumulating evidences suggest that chronic prostate inflammation may contribute to prostate cancer development and metastasis [17-20]. IL-6 is found to be produced in prostate cancer and stromal cells [21,22]. Increased level of serum IL-6 is correlated with poor prognosis and degree of metastasis in patient with prostate cancer [23]. IL-6 has been reported to facilitate prostate cancer progression and 


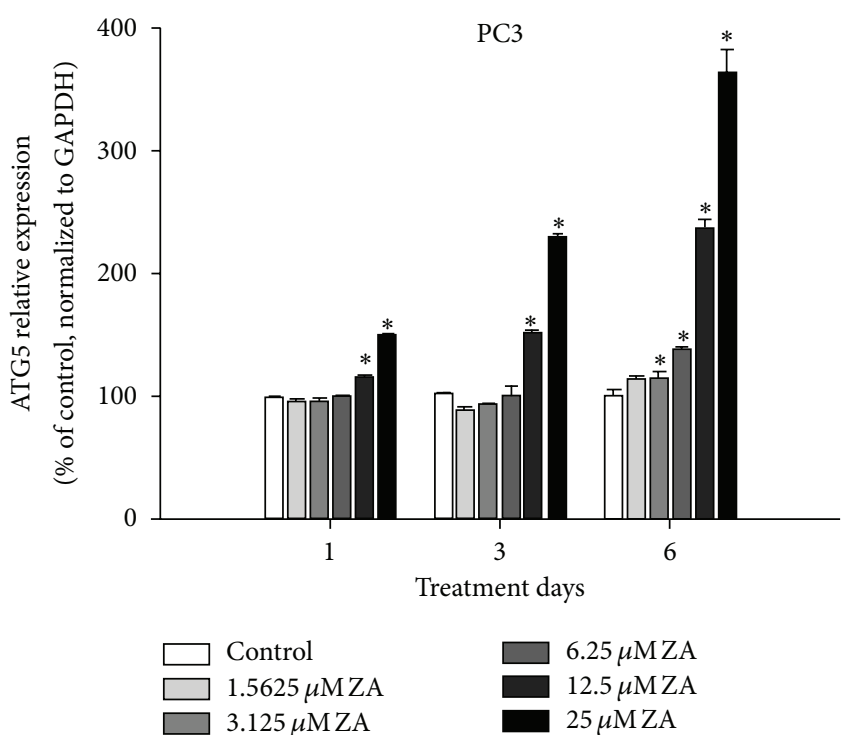

(a)

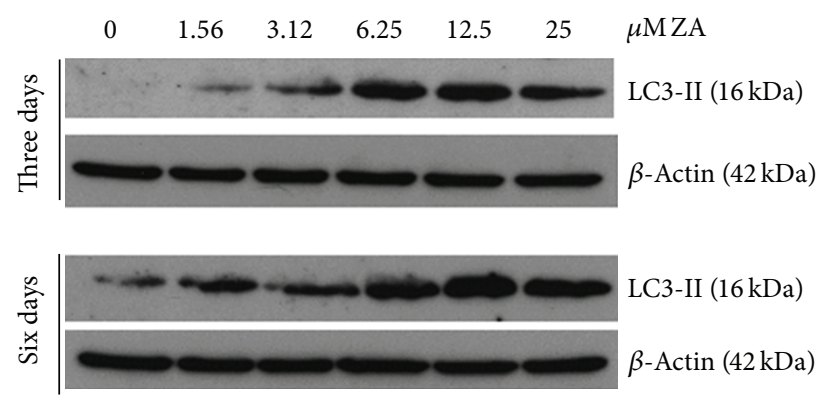

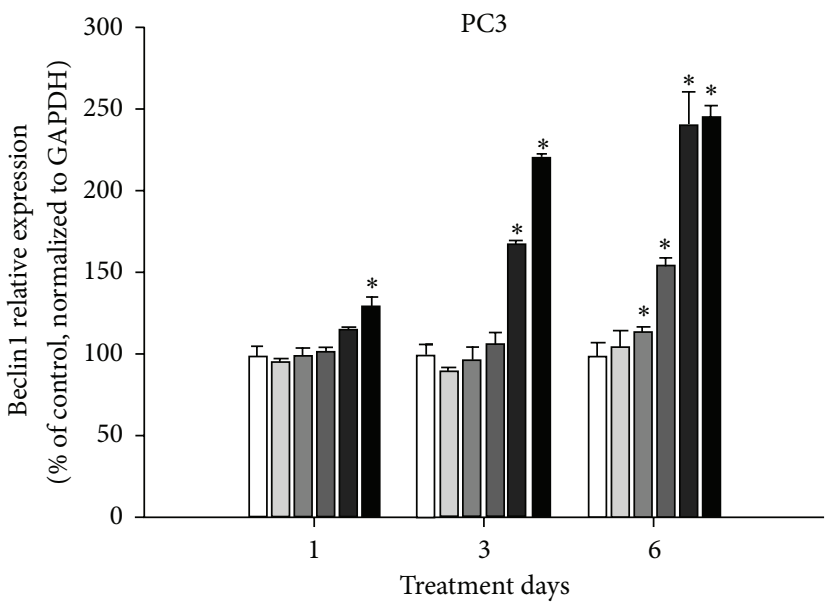

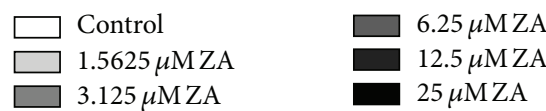

(b)

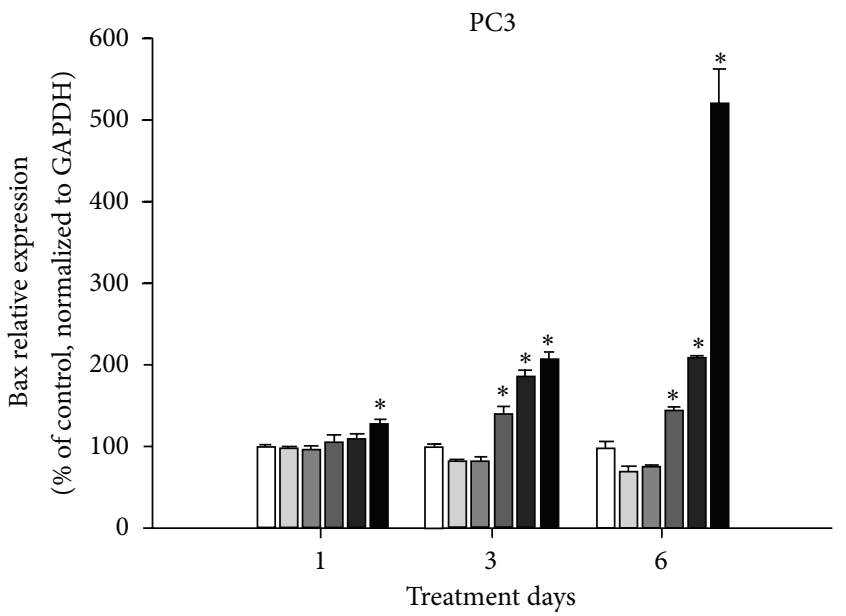

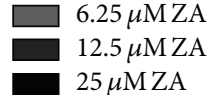

(d)

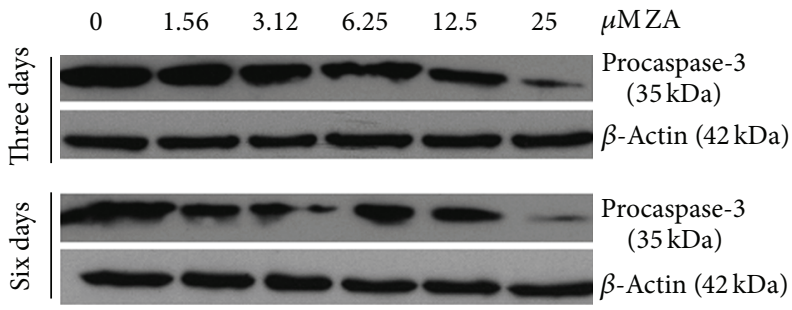

(e)

FIGURE 2: Effect of ZA on apoptotic and autophagy markers. PC3 cells were treated with ZA at indicated concentration $(0 \sim 25 \mu \mathrm{M})$ for 1 , 3, and 6 days. After treatment, total RNA was extracted and cDNA was reverse-transcribed from mRNA for real-time PCR of ATG5 (a), Beclin1 (b), and Bax (d). Total protein was extracted from cells to perform western blot. LC3-II antibody was used to determine the level of autophagosome formation (c). Procaspase-3 antibody was used to measure the activation of caspase- 3 (e). Data are expressed as mean \pm SD. ${ }^{*} P<0.05$ compared with control. 


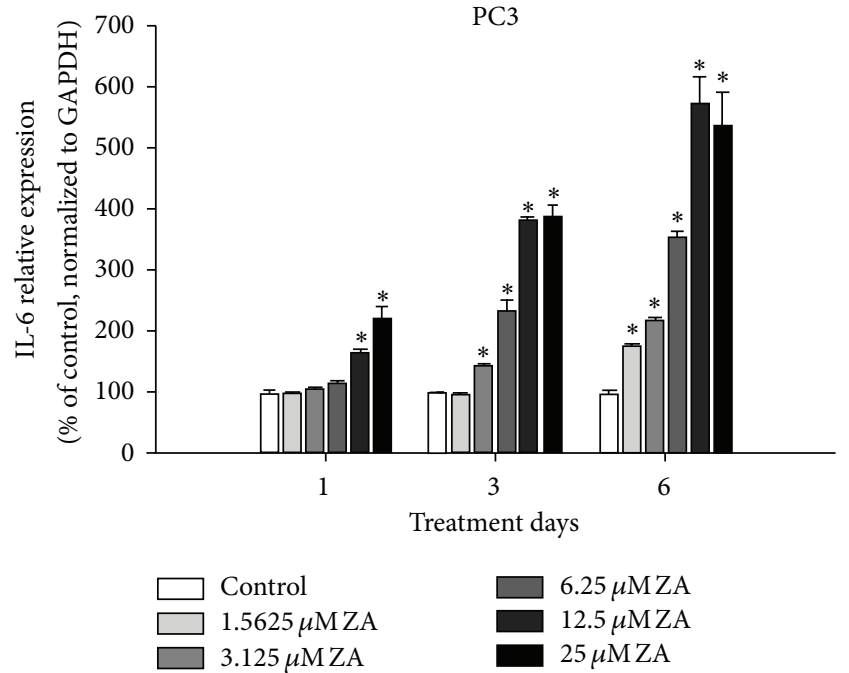

(a)

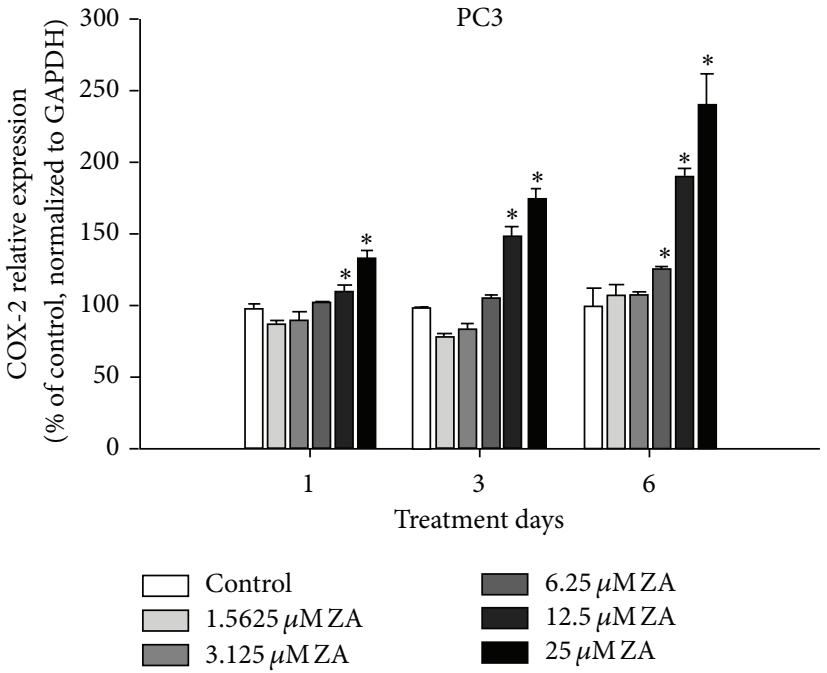

(b)

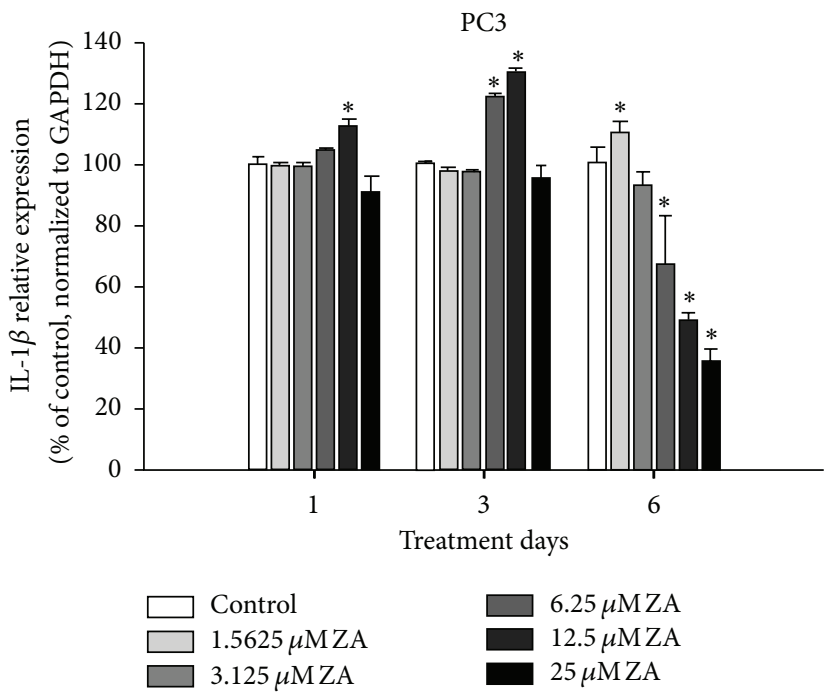

(c)

FIGURE 3: Effect of ZA on mRNA expression of inflammatory mediators. PC3 cells were treated with ZA at indicated concentration $(0 \sim 25 \mu \mathrm{M})$ for 1,3, and 6 days. After treatment, total RNA was extracted and cDNA was reverse-transcribed from mRNA for real-time PCR of IL-6 (a), COX-2 (b), and IL-1 $\beta$ (c). Experiments were performed in triplicate and the data were expressed as mean \pm SD. ${ }^{*} P<0.05$ compared with control.

bone metastasis through autocrine and paracrine mechanisms [24-26]. IL-6 enhances prostate cancer proliferation through activation of STAT, MAPK, and PI3K pathways; IL-6 also stimulates Janus kinase (JAK)/STAT3 pathways resulting in expression of RANKL and enhancement of osteoclastogenic activity [23, 27]. Manipulation of serum IL-6 has been reported to contribute to the management of prostate cancer $[28,29]$. We observed that PC3 cells exhibit high expression level of IL-6 upon ZA exposure in association with upregulation of NF- $\kappa$ B which initiates IL-6 production. The increased production of IL- 6 was accompanied with upregulated expression of antiapoptotic gene Bcl2. Interestingly, ZA treatment also induced apoptosis as a result of upregulated expression of apoptotic gene Bax. Our data indicate that treatment of PC3 cells with ZA leads to simultaneous induction of apoptosis and survival signaling. The finding may be in support of a recent in vivo study in which ZA fails to prevent bone-metastasis development in prostate cancer [30]. It is suggested that apoptotic effect of ZA may be indirectly compensated by coexpression of IL- 6 through autocrine mechanism.

COX-2 is an enzyme catalyzing biosynthesis of prostaglandin that participates in a variety of physiological activity. The excessive expression of COX-2 is reported in many malignancies including prostate cancer, resulting in abundance of its enzymatic product prostaglandin E2 


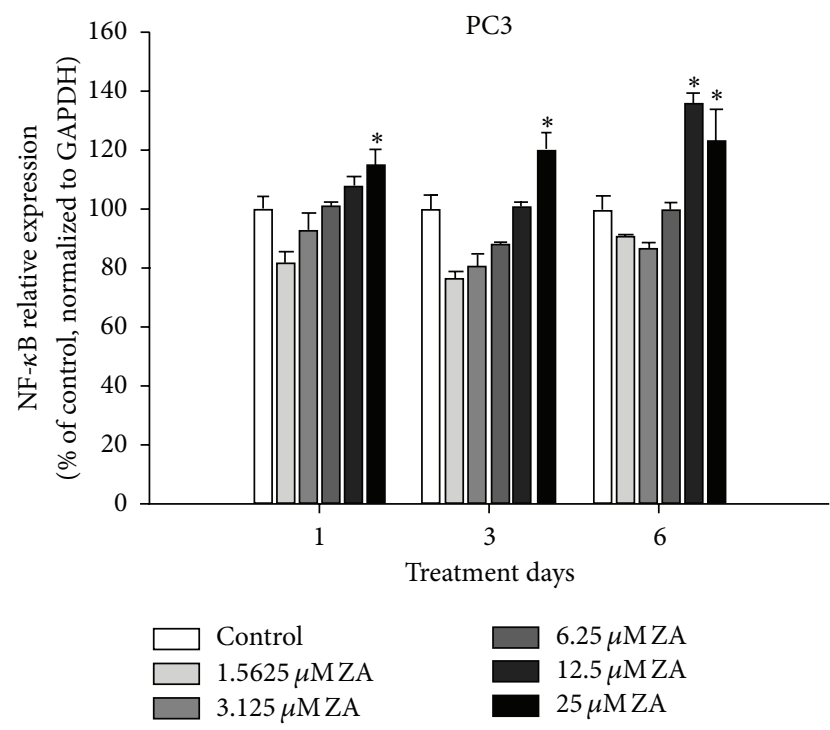

FIgURE 4: Effect of ZA on NF- $\kappa$ B mRNA expression. PC3 cells were treated with $\mathrm{ZA}$ at series concentration $(0 \sim 25 \mu \mathrm{M})$ for indicated days. After treatment, the mRNA expression of NF- $\kappa \mathrm{B}$ in ZAtreated PC3 cells was measured by real-time PCR. The expression of GAPDH was used as internal loading control. Experiments were performed in triplicate and the data were expressed as mean \pm SD. ${ }^{*} P<0.05$ compared with control group.

(PGE2) [31]. It is accepted that aberrant expression of COX-2 and upregulation of COX-2/PGE2 pathway play a critical role in carcinogenesis and tumorigenesis. Preclinical and clinical studies have shown that inhibition of COX-2 production reduces risk of prostate cancer and precancerous lesions [3234]. The PC3 cells treated with ZA exhibited an upregulation of mRNA expression of COX-2. Our data reveal that the increased COX-2 expression was associated with parallel enhancement of IL-6 secretion in PC3 cells. It is implied that COX-2 mediated production of IL-6 may synergistically contribute to the prosurvival of PC3 cells through induction of $\mathrm{Bcl}-2$ expression. Moreover, elevated COX-2 expression may consequently contribute to IL-6 production through a NF- $\kappa \mathrm{B}$-independent pathway. Unexpectedly, the level of IL- $1 \beta$ that theoretically activates COX-2 expression was decreased upon incubation of PC3 cells with ZA for 6 days. Further investigations are required to elucidate the mechanism by which IL- $1 \beta$ is reduced in the experimental setting.

Besides being capable of inducing inflammation responses, our data show that ZA exerts inhibitory effects on proliferation PC3 cells. It has been reported that ZA suppresses growth of prostate cancer cells through apoptosis or autophagy $[12,35]$. Our findings are in agreement with previous evidences and demonstrate that ZA induces both apoptosis and autophagy in PC3 cells. The expression of the inflammatory cytokines might attenuate the anticancer effects of zoledronic acid through auto- or paracrine effects. This phenomenon might partially explain the possibility of the absence of clinical elongation of survival in patients

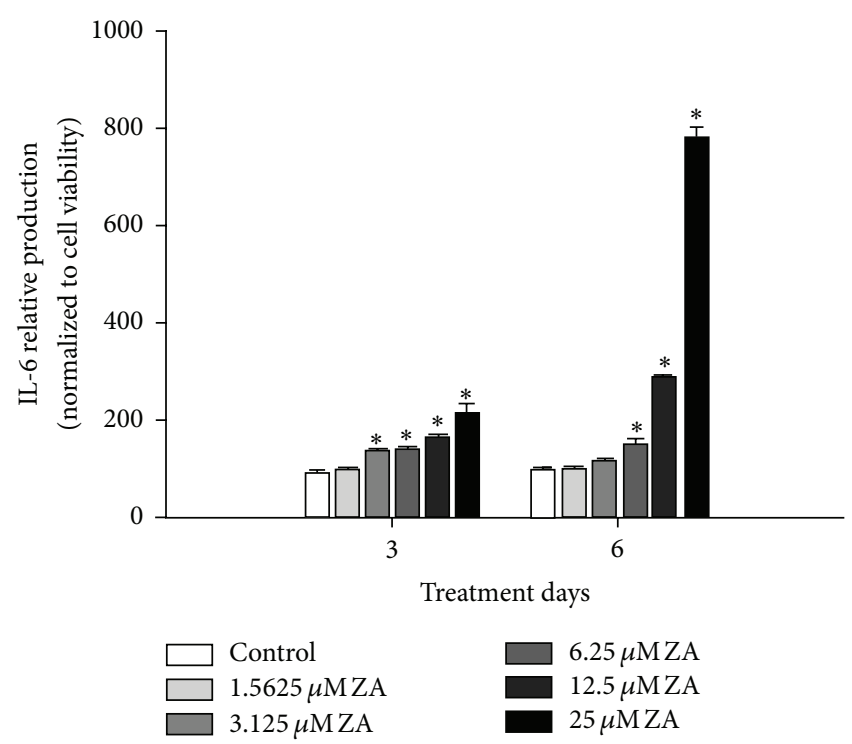

FIGURE 5: Effect of ZA on IL-6 production. Culture medium was collected from PC3 cells treated with series concentration of ZA $(0 \sim 25 \mu \mathrm{M})$ for indicated days. The protein concentration of IL- 6 in the medium was detected by ELISA as described in Section 2. Experiments were performed in triplicate and the data were expressed as mean \pm standard deviation. ${ }^{*} P<0.05$ compared with control.

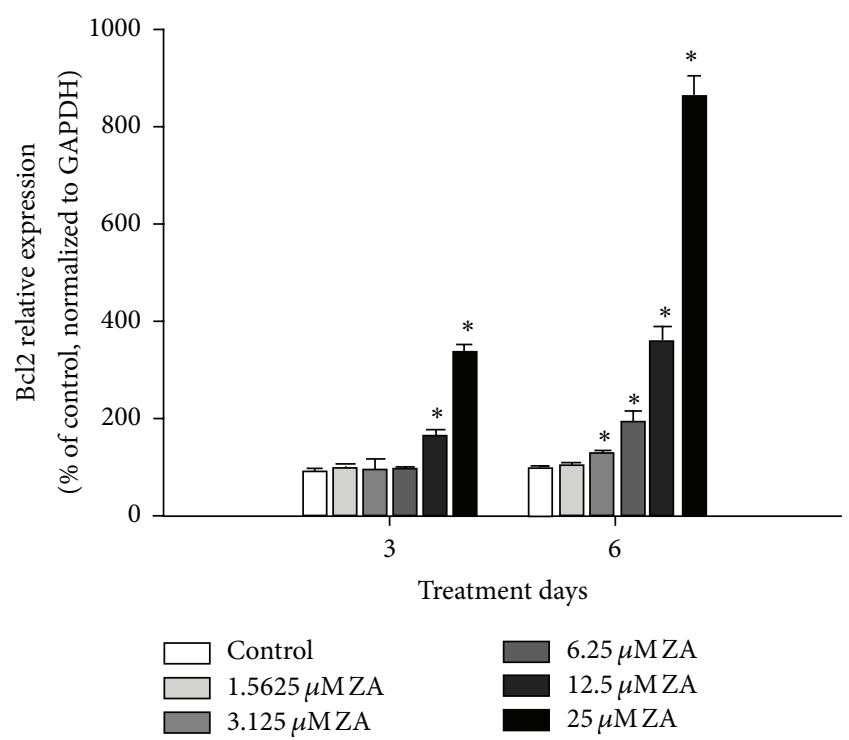

Figure 6: Effect of ZA on Bcl2 mRNA expression. PC3 cells were treated with ZA at series concentration $(0 \sim 25 \mu \mathrm{M})$ for indicated days. The mRNA expression of prosurvival $\mathrm{Bcl} 2$ was accessed by real-time PCR. GAPDH was used as internal control. Experiments were performed in triplicate and the data were expressed as mean \pm standard deviation. ${ }^{*} P<0.05$ compared with control.

on zoledronic acid. Besides, these results may implicate the possibility of combined therapies using ZA with anti-inflammatory agents to improve its anticancer effects. 


\section{Conclusions}

ZA induced cell death of prostate cancer PC3 cells through activation of apoptosis and autophagy while treating in low dose. Activation of inflammatory biomarkers including IL6 , COX-2, and NF- $\kappa \mathrm{B}$ was observed in ZA-treated PC3 cells. In pharmacokinetic aspect, ZA is gradually metabolized and subsequently exerts different pharmacological effects in different dosages. Our findings provoke a consideration that use of ZA for anticancer or antimetastasis regimen may have coexisting effects that compromise or abolish the intended beneficial effects against cancer.

\section{Conflict of Interests}

The authors confirm that the content of this study has no conflict of interests.

\section{Authors' Contribution}

Yi-Chia Lin and Thomas I-Sheng Hwang contributed equally to this work.

\section{Acknowledgment}

This study was supported by Shin Kong Wu Ho-Su Memorial Hospital (SKH-8302-100-0302 and SKH-8302-101-DR-06, to Yi-Chia Lin, and SKH-8302-97-DR-14 and SKH-8302-1000301, to Thomas I-Sheng Hwang).

\section{References}

[1] S. P. Luckman, D. E. Hughes, F. P. Coxon, R. G. G. Russell, and M. J. Rogers, "Nitrogen-containing bisphosphonates inhibit the mevalonate pathway and prevent post-translational prenylation of GTP-binding proteins, including Ras," Journal of Bone and Mineral Research, vol. 13, no. 4, pp. 581-589, 1998.

[2] S. C. L. M. Cremers, G. Pillai, and S. E. Papapoulos, "Pharmacokinetics/pharmacodynamics of bisphosphonates: use for optimisation of intermittent therapy for osteoporosis," Clinical Pharmacokinetics, vol. 44, no. 6, pp. 551-570, 2005.

[3] G. Morgan and A. Lipton, "Antitumor effects and anticancer applications of bisphosphonates," Seminars in Oncology, vol. 37, supplement 2, pp. S30-S40, 2010.

[4] I. R. Reid, G. D. Gamble, P. Mesenbrink, P. Lakatos, and D. M. Black, "Characterization of and risk factors for the acute-phase response after zoledronic acid," Journal of Clinical Endocrinology and Metabolism, vol. 95, no. 9, pp. 4380-4387, 2010.

[5] T. Tanvetyanon and P. J. Stiff, "Management of the adverse effects associated with intravenous bisphosphonates," Annals of Oncology, vol. 17, no. 6, pp. 897-907, 2006.

[6] G. S. Wilkinson, Y.-F. Kuo, J. L. Freeman, and J. S. Goodwin, "Intravenous bisphosphonate therapy and inflammatory conditions or surgery of the jaw: a population-based analysis," Journal of the National Cancer Institute, vol. 99, no. 13, pp. 1016-1024, 2007.

[7] C. M. Novince, B. B. Ward, and L. K. McCauley, "Osteonecrosis of the jaw: an update and review of recommendations," Cells Tissues Organs, vol. 189, no. 1-4, pp. 275-283, 2008.
[8] E. L. Scheller, K. D. Hankenson, J. S. Reuben, and P. H. Krebsbach, "Zoledronic acid inhibits macrophage SOCS3 expression and enhances cytokine production," Journal of Cellular Biochemistry, vol. 112, no. 11, pp. 3364-3372, 2011.

[9] P. Tsagozis, F. Eriksson, and P. Pisa, "Zoledronic acid modulates antitumoral responses of prostate cancer-tumor associated macrophages," Cancer Immunology, Immunotherapy, vol. 57, no. 10, pp. 1451-1459, 2008.

[10] E. Giraudo, M. Inoue, and D. Hanahan, "An amino-bisphosphonate targets MMP-9-expressing macrophages and angiogenesis to impair cervical carcinogenesis," Journal of Clinical Investigation, vol. 114, no. 5, pp. 623-633, 2004.

[11] J. C. Dumon, F. Journé, N. Kheddoumi, L. Lagneaux, and J. J. Body, "Cytostatic and apoptotic effects of bisphosphonates on prostate cancer cells," European Urology, vol. 45, no. 4, pp. 521$528,2004$.

[12] J. F. Lin, Y. C. Lin, Y. H. Lin et al., "Zoledronic acid induces autophagic cell death in human prostate cancer cells," Journal of Urology, vol. 185, no. 4, pp. 1490-1496, 2011.

[13] S. Gupta, M. Srivastava, N. Ahmad et al., "Over-expression of cyclooxygenase-2 in human prostate adenocarcinoma," Prostate, vol. 42, no. 1, pp. 73-78, 2000.

[14] R. Coleman, R. Cook, V. Hirsh, P. Major, and A. Lipton, "Zoledronic acid use in cancer patients," Cancer, vol. 117, no. 1, pp. 11-23, 2011.

[15] F. Saad, "Zoledronic acid: past, present and future roles in cancer treatment," Future Oncology, vol. 1, no. 2, pp. 149-159, 2005.

[16] J. R. Green, "Antitumor effects of bisphosphonates," Cancer, vol. 97, supplement 3, pp. 840-847, 2003.

[17] A. Bardia, E. A. Platz, S. Yegnasubramanian, A. M. de Marzo, and W. G. Nelson, "Anti-inflammatory drugs, antioxidants, and prostate cancer prevention," Current Opinion in Pharmacology, vol. 9, no. 4, pp. 419-426, 2009.

[18] J. Haverkamp, B. Charbonneau, and T. L. Ratliff, "Prostate inflammation and its potential impact on prostate cancer: a current review," Journal of Cellular Biochemistry, vol. 103, no. 5, pp. 1344-1353, 2008.

[19] S. Sutcliffe and E. A. Platz, "Inflammation in the etiology of prostate cancer: an epidemiologic perspective," Urologic Oncology: Seminars and Original Investigations, vol. 25, no. 3, pp. 242-249, 2007.

[20] A. M. de Marzo, E. A. Platz, S. Sutcliffe et al., "Inflammation in prostate carcinogenesis," Nature Reviews Cancer, vol. 7, no. 4, pp. 256-269, 2007.

[21] A. Shiirevnyamba, T. Takahashi, H. Shan et al., "Enhancement of osteoclastogenic activity in osteolytic prostate cancer cells by physical contact with osteoblasts," British Journal of Cancer, vol. 104, no. 3, pp. 505-513, 2011.

[22] C. Morrissey, J. S. Lai, L. G. Brown et al., "The expression of osteoclastogenesis-associated factors and osteoblast response to osteolytic prostate cancer cells," Prostate, vol. 70, no. 4, pp. 412424, 2010.

[23] K. Tawara, J. T. Oxford, and C. L. Jorcyk, "Clinical significance of interleukin (IL)-6 in cancer metastasis to bone: potential of anti-IL-6 therapies," Cancer Management and Research, vol. 3, no. 1, pp. 177-189, 2011.

[24] A. J. Costello, "Contemporary view of prostate cancer diagnosis and treatment," Australian Family Physician, vol. 32, no. 6, pp. 396-398, 2003. 
[25] N. M. Corcoran and A. J. Costello, "Interleukin-6: minor player or starring role in the development of hormone-refractory prostate cancer?” BJU International, vol. 91, no. 6, pp. 545-553, 2003.

[26] F. R. Santer, K. Malinowska, Z. Culig, and I. T. Cavarretta, "Interleukin-6 trans-signalling differentially regulates proliferation, migration, adhesion and maspin expression in human prostate cancer cells," Endocrine-Related Cancer, vol. 17, no. 1, pp. 241-253, 2010.

[27] T. Ara and Y. A. Declerck, "Interleukin-6 in bone metastasis and cancer progression," European Journal of Cancer, vol. 46, no. 7, pp. 1223-1231, 2010.

[28] B. Paule, S. Terry, L. Kheuang, P. Soyeux, F. Vacherot, and A. de Taille, "The NF- $\kappa \mathrm{B} / \mathrm{IL}-6$ pathway in metastatic androgenindependent prostate cancer: new therapeutic approaches?" World Journal of Urology, vol. 25, no. 5, pp. 477-489, 2007.

[29] Z. Culig, H. Steiner, G. Bartsch, and A. Hobisch, "Interleukin6 regulation of prostate cancer cell growth," Journal of Cellular Biochemistry, vol. 95, no. 3, pp. 497-505, 2005.

[30] T.-T. Hung, J. Chan, P. J. Russell, and C. A. Power, "Zoledronic acid preserves bone structure and increases survival but does not limit tumour incidence in a prostate cancer bone metastasis model," PLoS ONE, vol. 6, no. 5, Article ID e19389, 2011.

[31] E. Richardsen, R. D. Uglehus, J. Due, C. Busch, and L.-T. Busund, "COX-2 is overexpressed in primary prostate cancer with metastatic potential and may predict survival. A comparison study between COX-2, TGF- $\beta$, IL-10 and Ki67," Cancer Epidemiology, vol. 34, no. 3, pp. 316-322, 2010.

[32] R. E. Harris, "Cyclooxygenase-2 (cox-2) blockade in the chemoprevention of cancers of the colon, breast, prostate, and lung," Inflammopharmacology, vol. 17, no. 2, pp. 55-67, 2009.

[33] G. A. Gallego, S. D. Prado, P. J. Fonseca, R. G. Campelo, J. C. Espinosa, and L. M. A. Aparicio, "Cyclooxygenase-2 (COX-2): a molecular target in prostate cancer," Clinical and Translational Oncology, vol. 9, no. 11, pp. 694-702, 2007.

[34] T. Takahashi, H. Uehara, Y. Bando, and K. Izumi, "Soluble EP2 neutralizes prostaglandin E2-induced cell signaling and inhibits osteolytic tumor growth," Molecular Cancer Therapeutics, vol. 7, no. 9, pp. 2807-2816, 2008.

[35] H. L. Neville-Webbe, M. Gnant, and R. E. Coleman, "Potential anticancer properties of bisphosphonates," Seminars in Oncology, vol. 37, supplement 1, pp. S53-S65, 2010. 


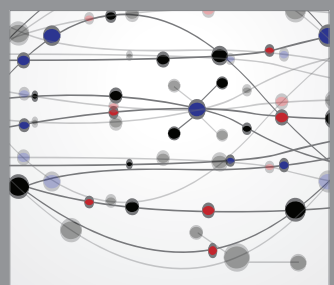

The Scientific World Journal
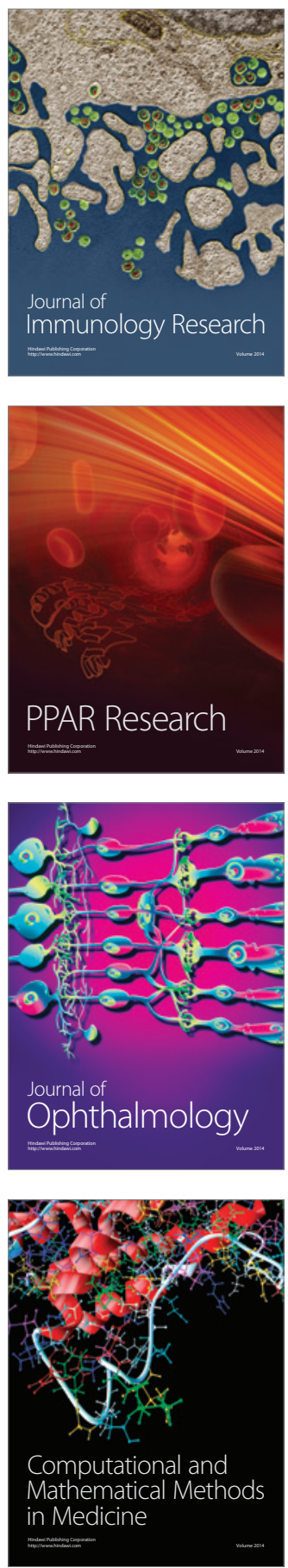

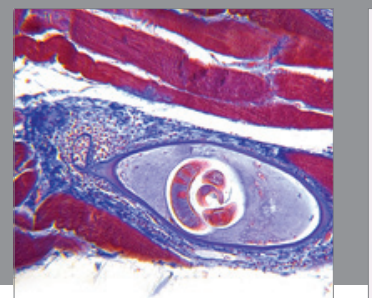

Gastroenterology

Research and Practice
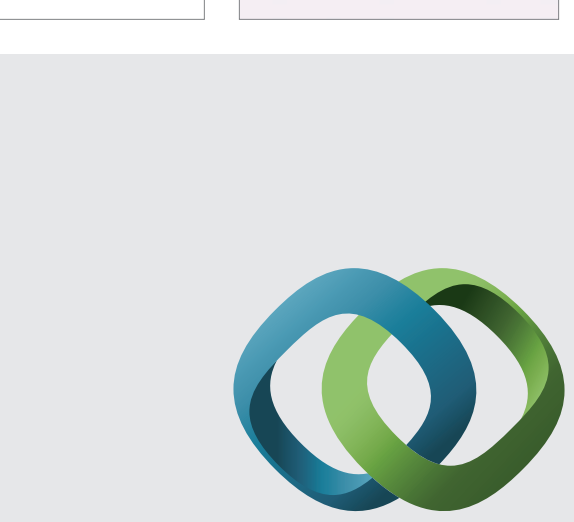

\section{Hindawi}

Submit your manuscripts at

http://www.hindawi.com
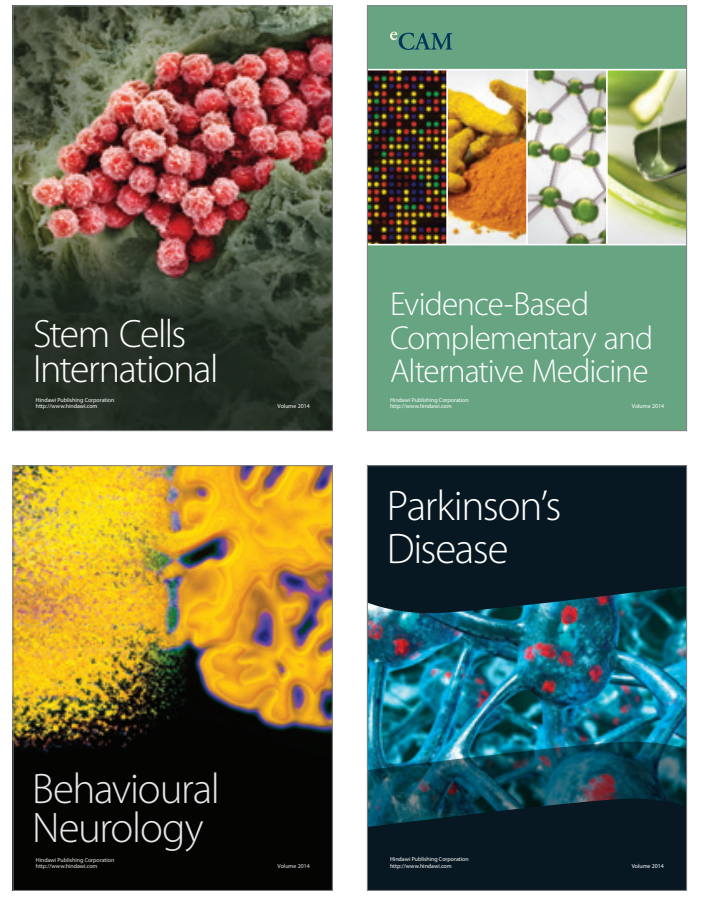
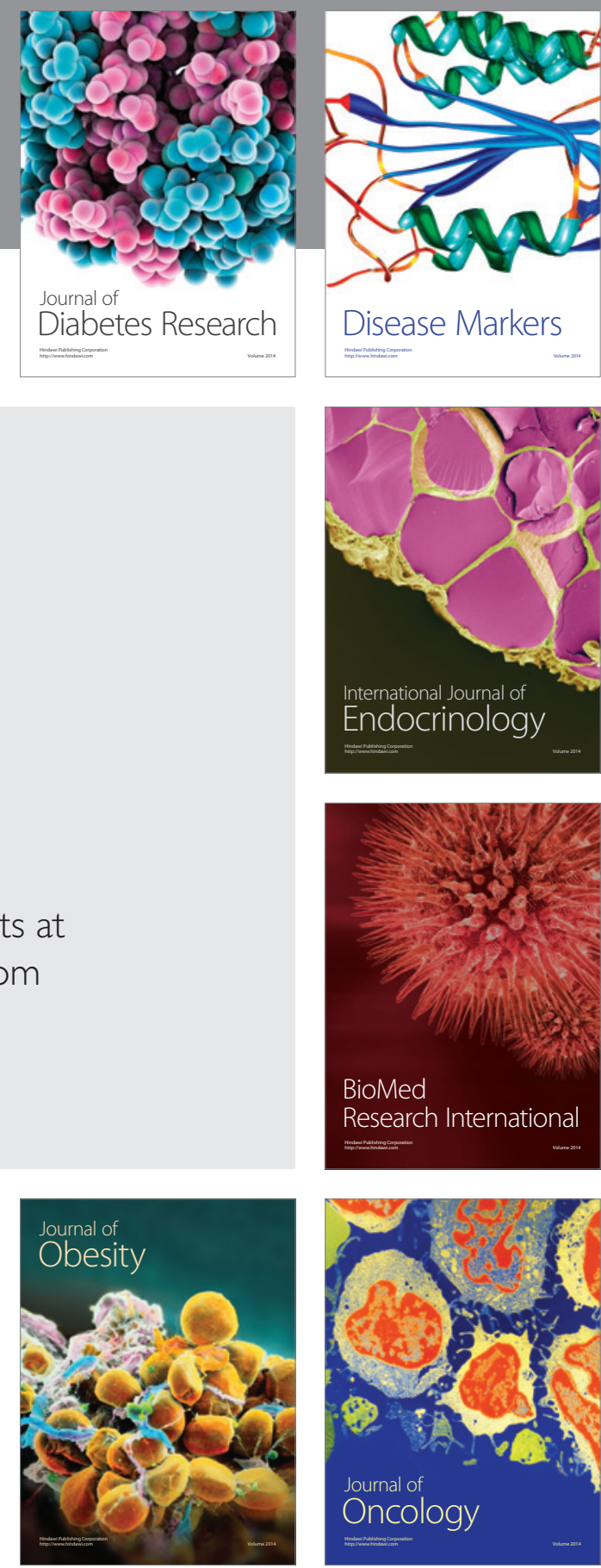

Disease Markers
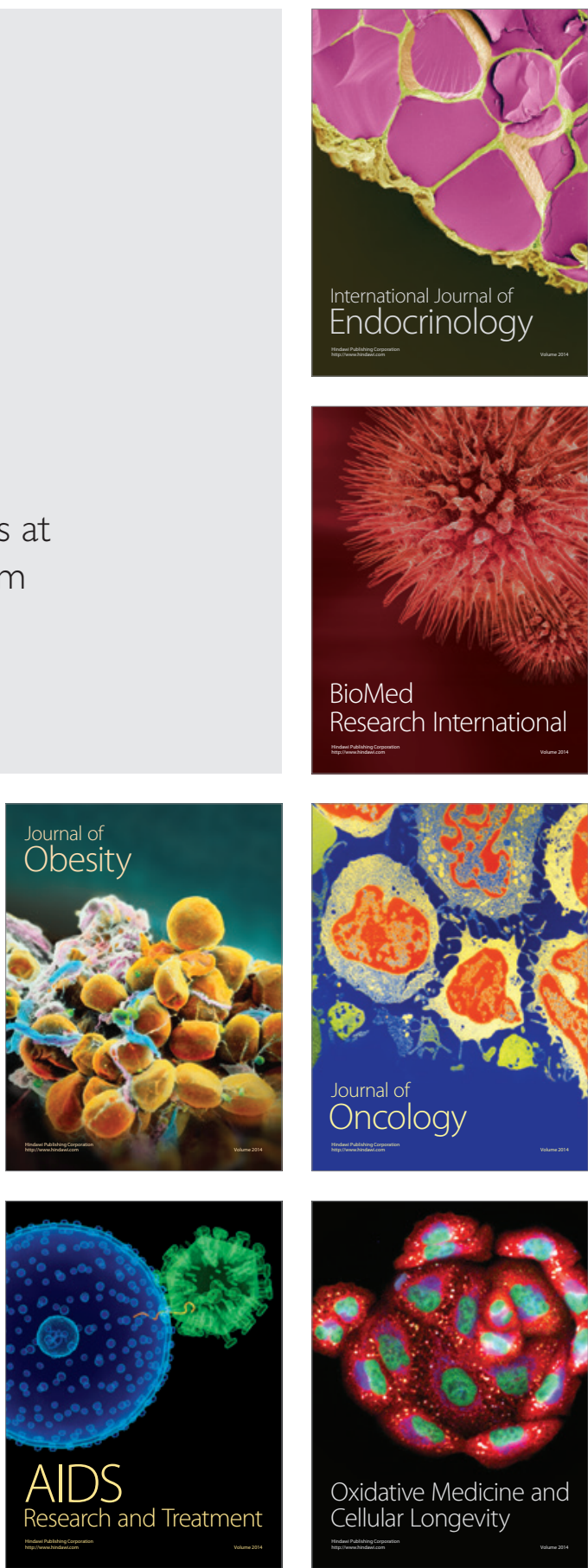